\title{
Video Article \\ BtM, a Low-cost Open-source Datalogger to Estimate the Water Content of Nonvascular Cryptogams
}

\author{
María Leo* ${ }^{1}$, Angel Lareo* ${ }^{2}$, Carlos Garcia-Saura ${ }^{2}$, Joaquín Hortal ${ }^{3,4}$, Nagore G. Medina ${ }^{5}$ \\ ${ }^{1}$ Real Jardín Botánico (CSIC-RJB) \\ ${ }^{2}$ Grupo de Neurocomputación Biológica, Dpto. de Ingeniería Informática, Escuela Politécnica Superior, Universidad Autónoma de Madrid \\ ${ }^{3}$ Department of Biogeography and Global Change, Museo Nacional de Ciencias Naturales (MNCN-CSIC) \\ ${ }^{4}$ Centre for Ecology, Evolution and Environmental Changes (cE3c), Faculdade de Ciências da Universidade de Lisboa \\ ${ }^{5}$ Department of Botany, Faculty of Science, University of South Bohemia \\ * These authors contributed equally
}

Correspondence to: María Leo at maria.leomontes@gmail.com, Angel Lareo at angel.lareo@uam.es

URL: https://www.jove.com/video/58700

DOI: doi: $10.3791 / 58700$

Keywords: Environmental Sciences, Issue 145, conductance measurement, cryptogam, water content, bryophyte, lichen, conductivity, impedance Date Published: $3 / 25 / 2019$

Citation: Leo, M., Lareo, A., Garcia-Saura, C., Hortal, J., Medina, N.G. BtM, a Low-cost Open-source Datalogger to Estimate the Water Content of Nonvascular Cryptogams. J. Vis. Exp. (145), e58700, doi:10.3791/58700 (2019).

\section{Abstract}

Communities of nonvascular cryptogams, such as mosses or lichens, are an important part of the Earth's biodiversity, contributing to the regulation of the carbon and nitrogen cycles in many ecosystems. Being poikilohydric organisms, they do not actively control their internal water content and need a humid environment to activate their metabolism. Therefore, studying water relationships of nonvascular cryptogams is crucial to understand both their diversity patterns and their functions in the ecosystems. We present the BtM datalogger, a low-cost open-source platform for the study of the water content of nonvascular cryptogams. The datalogger is designed to measure ambient temperature, humidity, and conductance from up to eight samples simultaneously. We provide a design for a printed circuit board (PCB), a detailed protocol to assemble the components, and the required source code. All this makes the assembly of the BtM datalogger accessible to any research group, even to those without previous specialized knowledge. Therefore, the design presented here has the potential to help popularize the use of this type of device among ecologists and field biologists.

\section{Video Link}

The video component of this article can be found at https://www.jove.com/video/58700/

\section{Introduction}

Communities of nonvascular cryptogams are a ubiquitous and an often-neglected part of terrestrial ecosystems ${ }^{1}$. They are made up of an aggregate of very different small-sized organisms among which bryophytes and lichens are the outstanding primary producers. These two groups of organisms share a physiologic characteristic that makes them unique: poikilohydry, or the inability to actively control their internal water content. This has profound implications for their physiological processes since the metabolism ceases when the cells are dried out in response to low levels of humidity and resumes when the environment is humid again ${ }^{2}$. As a consequence, nonvascular cryptogams avoid drought instead of coping with $\mathrm{it}^{2}$, which allows these communities to survive in a wide range of environments from cold and hot deserts to the tropics ${ }^{3,4}$.

Besides, they also show relatively simple structures and have low nutrient requirements. These characteristics make them highly sensitive to microclimatic conditions. In fact, nonvascular cryptogams often occupy a niche space that is unavailable to vascular plants of a larger size, forming ecosystems in miniature that constitute an important part of the world's diversity. Bryophytes and lichens alone include almost 40,000 species (ca. 20,000 bryophytes sensu lato ${ }^{5,6}$ and ca. 20,000 lichens ${ }^{7}$ ). Furthermore, their contribution to the Earth's biodiversity is even larger since their communities offer shelter for a vast number of species of fungi, including a diverse flora of free-living and mycorrhizal fungi, $\mathrm{N}$-fixating cyanobacteria growing as epiphytes, and a myriad of micro-invertebrates, such as tardigrades, collembola, myriapods, insects, and mites that take advantage of the water retention capacity and buffered conditions inside these miniature ecosystems.

Communities of non-vascular cryptogams also contribute to the regulation of biogeochemical carbon cycles. In dry ecosystems, the so-called biological soil crusts cover up to $40 \%$ of their surface ${ }^{8}$ and play a major role as carbon sinks. A recent review estimated that biological soil crusts of dry environments could be fixing $7 \%$ of all carbon fixed by terrestrial vegetation. Besides, in other ecosystems where either bryophytes or lichens or a combination of both are the primary producers - like some boreal forest systems or peat bogs - they produce between $30 \%$ and $100 \%$ of the total net primary productivity ${ }^{10,11}$. They are also important in ecosystems in which these organisms are not dominant, such as temperate forests. Indeed, forest floor bryophytes had an annual carbon uptake equivalent of about $10 \%$ forest floor respiration in a New Zealand temperate rainforest. Further, they are also important for nitrogen fixation, since the cyanobacteria living as epiphytes in these communities could be fixating almost $50 \%$ of the global amount of biological nitrogen ${ }^{4}$. 
Due to the dependence of their physiologic activity on the availability of water in the surrounding environment, both the diversity of nonvascular cryptogam communities and their functions in the ecosystems are strongly dependent on water content ${ }^{2}$. Note that, since they cannot actively control the water content in their tissues, their roles in carbon balance and nitrogen fixation are coupled with the hydration and desiccation cycles and, therefore, depend on the interval and periodicity of the dry-wet cycles. Thus, knowing the water content status of these organisms in realtime is key to understand the functions performed by cryptogams in the ecosystems.

Despite its importance, the development of methods to measure the water content and physiological activity in poikilohydric organisms has been relatively slow. In 1991 , Coxson ${ }^{12}$ made a first approach to directly measure the water content of lichens. After that, there was a gap in this kind of study until a recent development, when several works have provided methods to approximate measures of the physiologic status of nonvascular cryptogams ${ }^{13,14,15,16}$. Nevertheless, such knowledge is still scarce and scattered, and these works are mostly focused on soil crusts $^{4,8}$. However, bryophytes and lichens also play a relevant role in many other ecosystems, particularly at temperate, boreal, and polar regions ${ }^{1}$, and their importance is significant not only in soil communities but also for epiphytic communities growing on trees and saxicolous communities on rocks. This lack of research is partially linked to the absence of commercially available measurement dataloggers, which forces research groups to build their own equipment. Developing a datalogger requires specific knowledge that most ecologists do not have, so it substantially increases the cost of implementing the relatively large measuring networks needed to gather representative data on the performance of nonvascular cryptogams along environmental and habitat gradients.

In this paper, we present a simple and cost-effective method to build a datalogger capable of measuring the conductance of nonvascular cryptogamic organisms simultaneously with the ambient temperature and humidity. It is programmed to record autonomously for relatively extended periods of time (up to two months) and is rugged enough to withstand harsh outdoor field conditions. Due to its simplicity, it will be a useful tool for ecologists and field biologists without specialized training in the development of dataloggers or those research groups that lack specialized staff. Therefore, this datalogger has the potential to help popularize the use of this type of device.

We developed a low-power and low-cost datalogger able to measure the conductance from up to eight different sources and record the environmental temperature and relative humidity simultaneously. The device is designed after Coxson's design ${ }^{12}$ and implemented on an opensource platform (Table of Materials). The aim was to prioritize the ease of assembly and power efficiency and to facilitate the maintenance of long-term installations. The design is derived from an article by Open Source Building Science Sensors (OSBSS) ${ }^{17}$. This design was modified by incorporating additional circuitry to read out the impedance of cryptogams and making it more compact and easier to manufacture.

The result is BtM board (Bryolichen Temperature Moisture board), an open-source printed circuit board ${ }^{18}$. Each board is controlled by a high energy-efficient microcontroller (Table of Materials). Environmental temperature and relative humidity data are gathered through a temperature and humidity sensor that comes precalibrated and, aside from its low power consumption, has an adequate price-performance ratio.

The board uses a digital communication protocol (standard SPI serial) to manage the measurement cycle. A real-time clock (DS3234) mounted on each board provides accurate timing. In order to reduce energy consumption, the processor remains in standby mode most of the time. Each time data needs to be collected, the real-time clock activates the processor and triggers the logging process. The real-time clock is also used to accurately record the date and time of each data case.

Up to eight moss and/or lichen samples can be logged in parallel using a single BtM board. When the experiment is set up, two crocodile-clip electrode probes are applied to each moss/lichen sample. Then, a voltage divider between each electrode and a resistor reference with a known value ( $330 \mathrm{~K} \Omega$ in this case) are used. This resistor value was selected through calibration and based on previous measures of the cryptogams. It provides a resolution of one order of magnitude around the reference value $(100-1,000 \mathrm{~K} \Omega)$. The voltage drop is buffered and then read with the microcontroller using its analog ports $(\mathrm{A} 0-\mathrm{A} 7)^{18}$. The voltage is calculated by applying the following formula.

$\mathrm{Vi}=(\mathrm{ADCi} \times \mathrm{VCC}) / 1023$

Here, $A D C i$ is the raw value from the ADC (Analog-to-Digital converter) of channel $i, V C C$ is the power supply voltage ( $3.3 \mathrm{~V}$ in this case), and 1023 is the range of the ADC output. The resulting voltage $\mathrm{Vi}$ is then used in combination with Ohm's law to calculate the resistance (Ri, $\Omega$ ) and conductance $(G, S)$ of each moss sample.

$\mathrm{Ri}=(\mathrm{VCC} \times \mathrm{RL}) / \mathrm{Vi}-\mathrm{RL}$

$G=1 / R i$

Here, $\mathrm{RL}$ is the value of the resistor reference ( $330 \mathrm{~K} \Omega$ in this case). The microcontroller's onboard software incorporates all these equations, so it can directly register the values of resistance and conductance.

The board also collects measurements of the ambient temperature and humidity using sensors. Then, each data point is written to a log file on a microSD card. A microSD TransFlash breakout board was mounted on each BtM board for this purpose. Finally, the microSD card can be manually collected after the experiment. All data points can be transferred to a computer for further analysis.

\section{Assembly of the Datalogger}

1. Prepare a soldering iron and a spool of solder wire. Wait for the soldering iron to heat up and moisten the cleaning sponge.

2. Cut the pin header strips to the desired length and solder them into the sockets for the temperature and humidity sensor, the microcontroller, and the RTC clock and microSD breakout modules.

1. To solder, preheat the desired join with the tip of the soldering iron.

2. Then, apply a small amount of material from the solder wire, enough to fill up the junction. 
3. Finally, remove the soldering iron and wait for the junction to cool down.

3. Assemble the components to the circuit board using the same procedure as in step 1.2, following the markings of the PCB and the component references specified in the Table of Materials (see Figure 1 for an assembly scheme).

1. First, solder the resistors. Then, solder the sockets for the operational amplifiers, the SHT7X sensor, and the RTC clock and microSD breakout modules.

2. Next, solder the two transistors. The board also needs to be soldered now, using pin headers. Finally, solder the connectors to the board.

4. Solder the SHT7X humidity/temperature sensor into a pin header or extension cable to reinforce the leads.

5. Prepare a multimeter in the continuity testing or conductivity testing mode. Use the multimeter to verify that there are no short circuits between any of the pins or connections.

1. Doublecheck the positive and negative terminals of the power supply. Also, verify that each solder joint creates a stable connection between the component pins and the copper tracks of the circuit.

NOTE: This step is very important; do not skip it.

6. Connect the battery terminals and cable clips to the board using a screwdriver.

1. First, use any cutting tool to strip $\sim 4 \mathrm{~mm}$ of each wire end, exposing the conductive core. Next, introduce each cable into the appropriate terminal and tighten the screw with the Phillips screwdriver.

2. Ensure and doublecheck the correct polarity of the cables, especially those of the power supply. Test the strength of the connection by pulling the cables slightly, verifying that everything is firmly connected.

7. To further reduce power consumption, remove the power LED of the microcontroller board by either desoldering or cutting off the LED diode from the board.

8. Finally, mount the BtM board in a weatherproof enclosure to keep moisture away from the electronics.

1. Fit the enclosure with the battery pack, connecting it to the positive and negative terminals. Mount the humidity/temperature sensor outside of the box, leaving it connected to the BtM board.

2. Route the eight pairs of crocodile clips needed for conductance measurements to the outside of the weatherproof enclosure. Last, clip each moss strand with the crocodile clips.

\section{Loading the Software}

1. Download and install the integrated development environment (IDE) 1.0 .6 from the website ${ }^{19}$. The microcontroller used is an opensource physical computing platform and it comes with its own IDE. It is important to download the adequate version since there are known compatibility problems with some of the required libraries.

2. Download the necessary libraries from the GitHub repository ${ }^{18}$ : DS3234, DS3234lib3, PowerSaver, SdFat, and Sensirion.

3. Download the main source code for the datalogger from the GitHub repository ${ }^{18}$.

4. Open the clock.ino file to set up the current time and date. Edit the parameters for the function RTC.setDateTime with the current time and date using the following format:

RTC.setDateTime(DD,MM,YY,hh,mm,ss); // Date: DD/MM/YY hh:mm:ss

Here, DD is day, MM is month, $Y Y$ is year, hh is hour, $\mathrm{mm}$ is minutes, and ss is seconds.

5. Then, upload the clock program to the BtM board, plugging the USB-to-Serial adapter (FTDI breakout) into the microcontroller programming ports and using a mini-USB-to-USB cable to connect the board to the computer. Finally, first press Verify and, then, Upload in the IDE.

6. Open the datalog project in the IDE and modify the datalog.ino file. Set up the start time for the logger editing the following variables: int dayStart $=\mathrm{DD}$, hourStart $=\mathrm{hh}$, minStart $=\mathrm{mm}$

Here, DD is the number of the day, hh is the starting hour of the measurements, and $\mathrm{mm}$ the minute of the start.

NOTE: The code to set up a specific time should look like this:

RTC.setDateTime(DD,MM,YY,hh,mm,ss);// Date 01/12/17 12:00:00

7. Set the interval between measurements (in seconds) modifying the value of the variable interval.

\section{Set-up of the Measurement Probes}

1. Place the crocodile clips at a central position of the communities in the cases of bryophytes, fruticose lichens and foliose lichens (Figure 2). For fruticose lichens, attach the clips in the thallus and, for mosses, directly on the stem of an individual. In the case of foliose lichens, place the clips on the border of the thallus.

2. Keep a minimum distance of ca. $5 \mathrm{~mm}$ between electrodes. Ensure that the clips are not easily detached before starting measurements.

\section{Calibration for Conductance Measurements}

1. To ensure that the specimens are dry, perform the calibration at noon, on a day with low air relative humidity, preceded by at least one, and preferably two, dry days.

2. Select a community of moss or lichens that is healthy and well-structured.

3. Connect the datalogger to the moss or lichen, following the steps in section 3 of this protocol.

4. Start the measurements (turn on the datalogger) and leave the BtM board running for approximately 3 min to stabilize the recorded values.

5. Perform a precalibration test to estimate the amount of water required in each watering event. Connect the clips to the sample and add water until the conductance reaches a value that does not increase with the addition of water. This is the maximum conductance value of that sample. This value will be used to establish the watering steps of the calibration (see step 4.7.1).

6. Wait until the conductance measures return to the initial values (the samples are dry). 
7. Then, add water sequentially with a small spray.

1. Moisten the samples with a quantity of water equivalent to $1 / 10$ of the amount of water required to achieve the maximum conductance (see step 4.5) in the sample.

2. Wait until the moss or lichen fully absorbs the water and the conductance measurements are stable before watering again $(\sim 1 \mathrm{~min}$ between each watering event).

3. Repeat until the conductance reaches the maximum value (saturation) and the moss or lichen is fully hydrated

NOTE: Each calibration test should take around $15 \mathrm{~min}$, depending on the interval between the waterings, which should be 1 - 2 min.

8. After finishing the calibration, take the microSD card from the BtM board and copy the data file to a computer.

NOTE: The logged values can then be used as a baseline for the experiments. It is also necessary to do this step to verify that the set-up is correctly registering the conductance of the samples, just before running the actual experiment.

\section{Alternative Calibration for Lab Experiments}

1. Fully hydrate the community of moss or lichen until an excess of external water is observed. To ensure that the community is fully hydrated, keep the community moist for 30 min.

2. Connect the datalogger to the moss or lichen, following the steps in section 3 of this protocol.

3. Start the measurements and leave the BtM board running for approximately 3 min to stabilize the recorded values.

4. Wait until the conductance reaches the minimum value (desiccation) and the moss or lichen is no longer conducting electricity. NOTE: Each calibration could last at least $1 \mathrm{~h}$, but the duration is highly variable depending on the species. Measurements should be taken until a minimum conductance value is achieved.

\section{Representative Results}

We analyzed the changes in conductance in two species of mosses, Dicranum scoparium Hedw. and Homalothecium aureum (Spruce) H. Rob. (Figure 3), during the calibration process in lab conditions. Mats of the two mosses were kept for $24 \mathrm{~h}$ in silica gel and placed in an artificial substrate (i.e., wadding) that kept their original structure (Figure 2). Then, the samples were watered $15 x$ to $20 x$ with a spray in 1 min intervals. Each watering event consisted of ca. $0.1 \mathrm{~mL}$ of water. In both species, a high correlation between the water added and the sample conductance (D. scoparium $r_{\mathrm{S}}=0.88, p<0.001 ; H$. aureum $r_{\mathrm{s}}=0.87, p<0.001$ ) was observed. There was a high increase in the conductance (from $0 \%$ to $25 \%$ at least) just in the first water addition, and the measures reached their maximum conductance at $4 \mathrm{~mL}$ for $D$. scoparium and $10 \mathrm{~mL}$ for $\mathrm{H}$. aureum. It is important to remark that the relationship between the quantity of water and conductance is logarithmic. Therefore, the values of conductance need to be transformed to have a linear relationship between both variables, and their relationship should be modeled using nonlinear regression.

We found some variability among the samples (see the different colors in Figures $\mathbf{3 a}$ and $\mathbf{3 b}$ ), although all samples belonging to the same species drew a similar curve. The variation between samples can be attributed to differences in biomass and morphology of the patches. Samples in the field are very likely to show this type of variability, so taking several measures of each community type is recommended. Not surprisingly, the highest variability was found among species, since species differ in several fundamental traits (e.g., the aggregation of the mats or morphology). To control for intra- and interspecies variability, we recommend calibrating each clip until achieving the maximum conductance values and, then, rescaling the results for each clip so that the values go from 0 to 100 . Consider that absolute conductance values depend on the distance between clips and the basal conductance of the stems, so the values they provide are not directly comparable.

The amount of water added in each watering event of the calibration process is crucial and will strongly affect the results. Here, the aim was to have several watering events in the range of maximum accuracy of the BtM. We present an example of a calibration curve when too much water is added in each step (Figure 4). If the sample is overwatered in the first watering event, the increase in the conductance cannot be appreciated and the calibration will be inaccurate. This may lead to biases in the range where nonvascular cryptogams are active, which are the most interesting measurements taken with the BtM.

We also analyzed the desiccation curve of the same two species $(H$. aureum and $D$. scoparium), to provide an alternative calibration procedure. Mats of the two mosses were watered overnight to ensure they were fully saturated. Then, a representative stem of each mat was extracted and placed in a stable, controlled environment and the conductance was recorded continuously. As for the other calibration measure, the values of conductance need to be transformed to have a linear relationship between both variables, and their relationship should be modeled using nonlinear regression.

Figures $\mathbf{5 a}$ and $\mathbf{5 b}$ show the dessication curves of $H$. aureum and the $D$. scoparium variability among samples of the same species. The intraand interspecies variability found were quite large and, as in the other calibration procedure, could be attributed to differences in biomass and morphology of each stem. To control for it, we recommend performing at least three measurements per species. Absolute conductance values are not directly comparable in this calibration procedure, as they also depend on the distance between clips and basal conductance of the stems.

We present an example of field data after a rain event occurred between June $23-26,2014$. We show the daily variation in the percentage of conductance (Figure 6a), relative humidity (Figure 6b), and precipitation (Figure 6c) for one species of moss (Syntrichia ruralis (Hedw.) F. Weber \& D. Mohr). There was a strong relationship between the conductance of the moss, the precipitation events, and the relative humidity of the air. During the period analyzed, there were two peaks in the conductance and humidity as a consequence of two precipitation events. The first one occurred just before midnight of June 23 and the second one after the midday of June 24 . About $8 \mathrm{~h}$ after the first rain event, we observed a decline in the relative humidity of the air, followed by a sudden drop in the moss conductance that goes below $25 \%$. The second rain event was smaller and, consequently, produced a smaller peak in conductance. After this rain event, the moss did not dry out immediately but stayed hydrated while the humidity was above $75 \%$. 


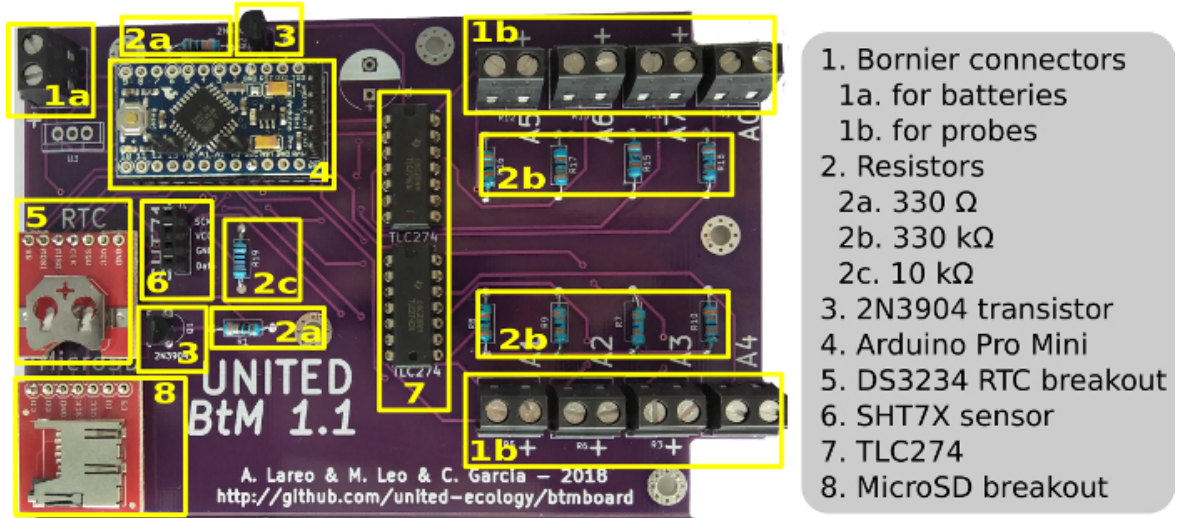

Figure 1: Assembly schematic of the BtM datalogger. The schematic includes a picture of the BtM board and the placement of each component on the board. Please click here to view a larger version of this figure.

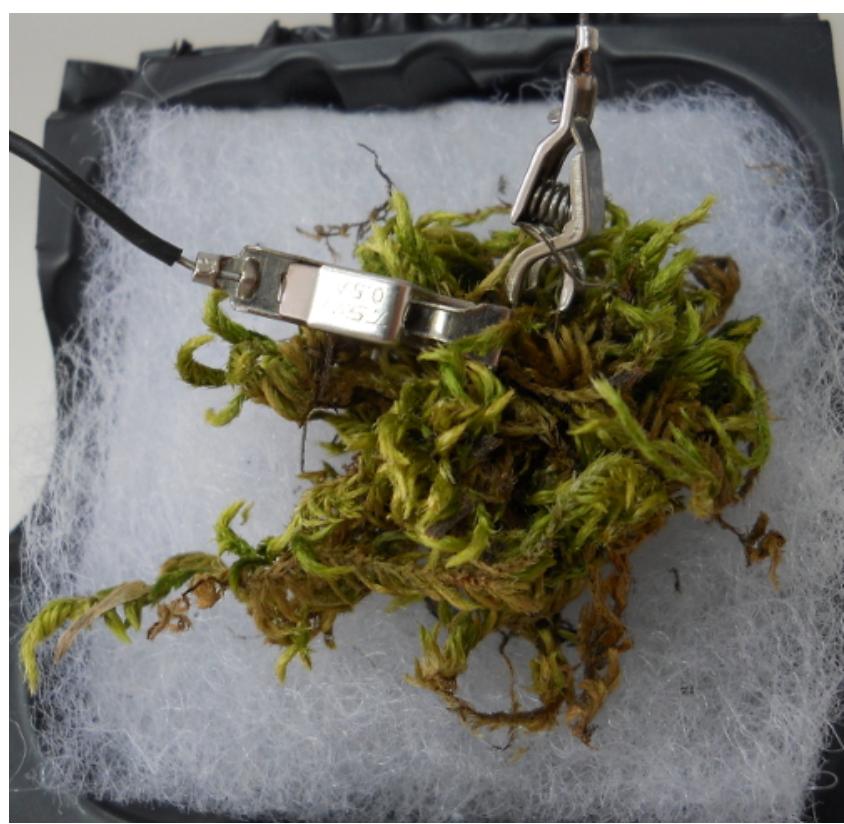

Figure 2: Correct placement of the clips in a moss (Homalothecium aureum). The image shows how to place the clips to maintain a minimal distance between the clips without damaging the bryophyte. Please click here to view a larger version of this figure.

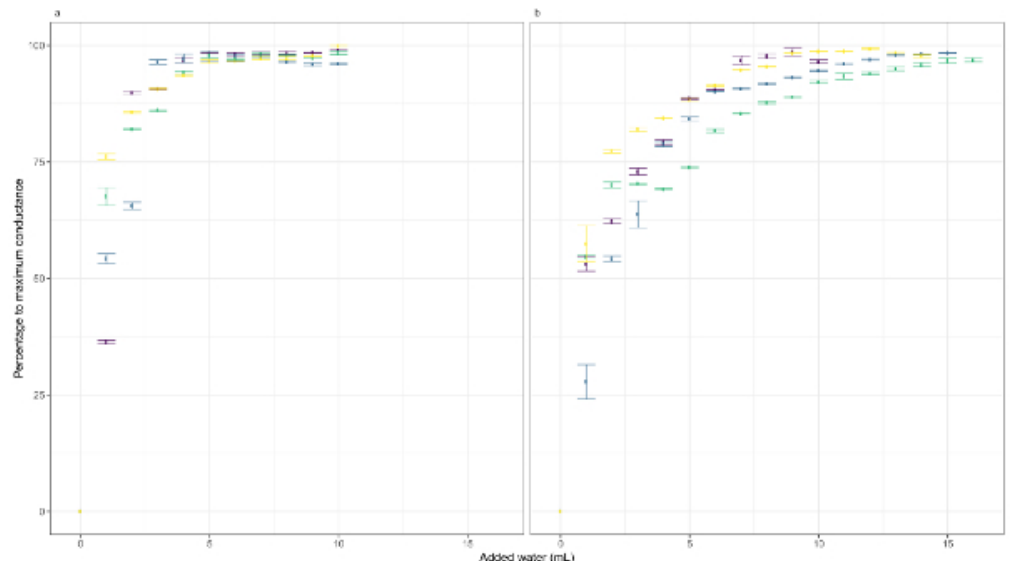

Figure 3: The response of conductance to water addition. These panels show the response of conductance to water addition in (a) Dicranum scoparium and (b) $\mathrm{H}$. aureum. The colors show the different replicates. The data points are the average of the log-transformed conductance in an interval between 10 and $30 \mathrm{~s}$ after the watering event. The error bars represent the standard deviation of the data in that interval. Please click here to view a larger version of this figure. 


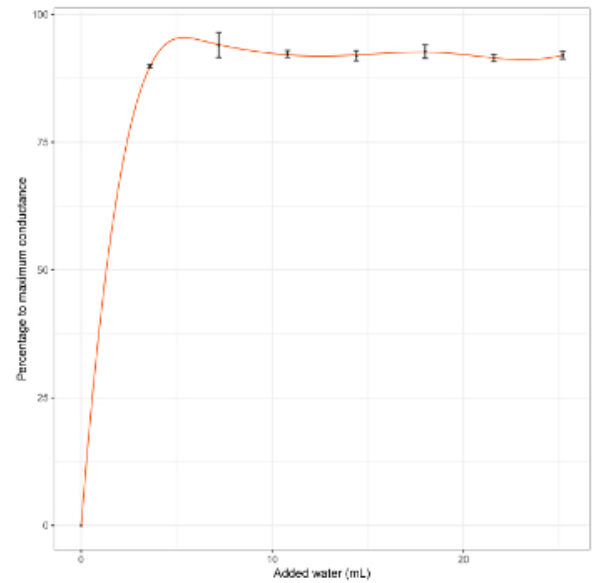

Figure 4: Response of log-transformed conductance to water addition in $D$. scoparium when the amount of added water is too large to allow calibration. The error bars represent the standard deviation of the data in that interval. Please click here to view a larger version of this figure.

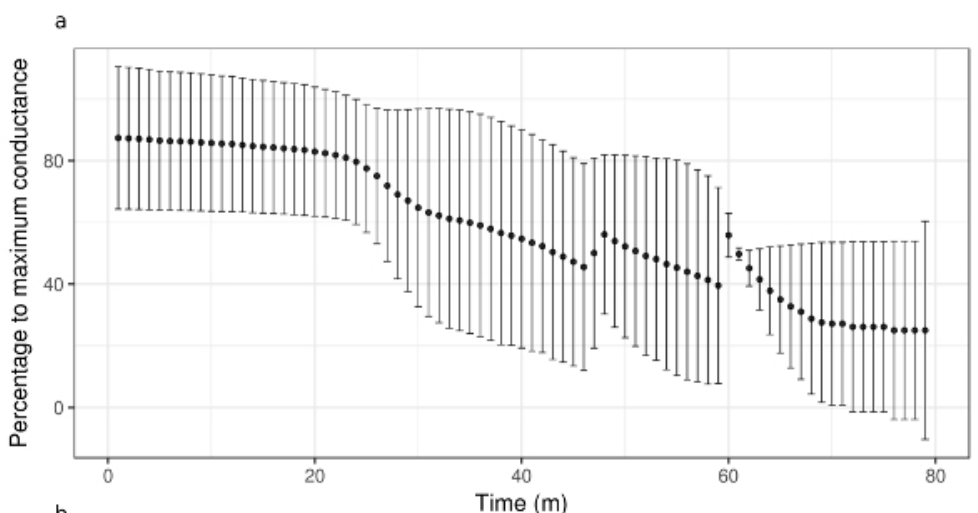

b

Time $(m)$

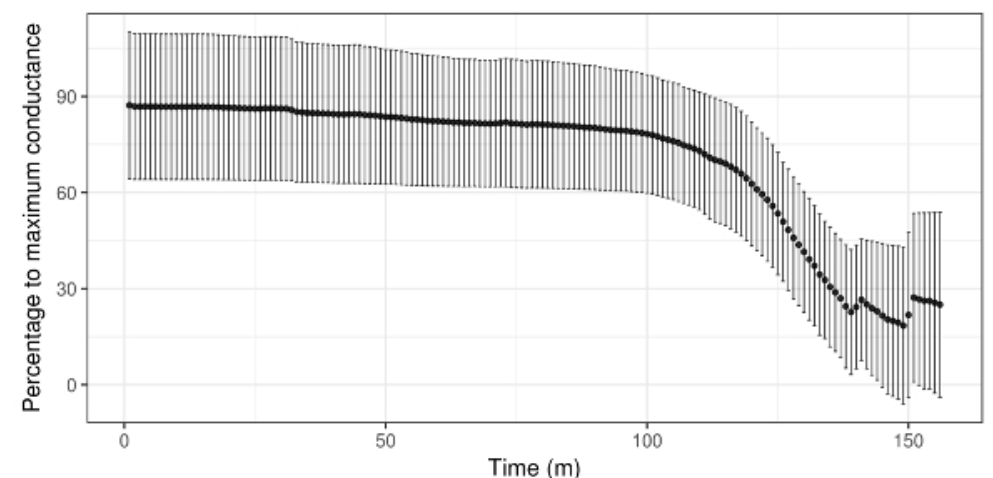

Figure 5: Desiccation curves. These panels show the desiccation curves of (a) $D$. scoparium and (b) $H$. aureum. The data points are the average of the log-transformed conductance measured every $30 \mathrm{~s}$. Black points show the mean of the three replicates and the error bars represent the standard deviation of the data in that interval. Please click here to view a larger version of this figure. 

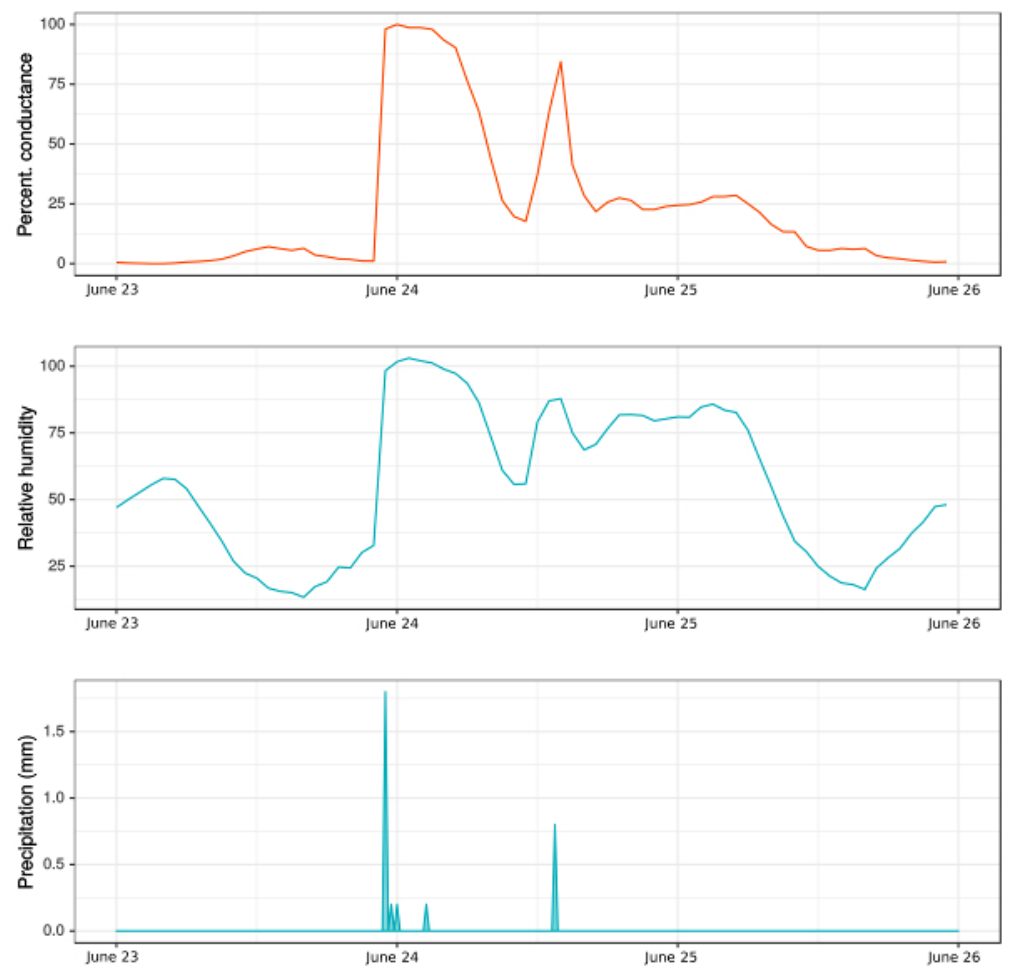

Figure 6: Daily variation in a moss' (Syntrichia ruralis) conductance, precipitation, and relative humidity. The measures were taken in soil communities of the Cantoblanco, Campus of the Universidad Autónoma de Madrid, Spain. The conductance and relative humidity were measured with the BtM prototype, while the precipitation data comes from a weather station placed a few meters away from the measurement location. Please click here to view a larger version of this figure.

\begin{tabular}{|l|l|l|l|}
\hline Date/Time & Temp(C) & RH(\%) & Conductance(KMho) \\
\hline $11 / 03 / 1812: 00$ & 26.6 & 66.6 & 139.53 \\
\hline $11 / 03 / 1812: 00$ & 26.6 & 66.4 & 167.92 \\
\hline $11 / 03 / 1812: 00$ & 26.8 & 66.4 & 199.14 \\
\hline $11 / 03 / 1812: 00$ & 26.9 & 66.4 & 212.75 \\
\hline $11 / 03 / 1812: 00$ & 26.6 & 66.6 & 217.15 \\
\hline $11 / 03 / 1812: 01$ & 26.9 & 66.7 & 218.93 \\
\hline $11 / 03 / 1812: 01$ & 27 & 66.8 & 139.53 \\
\hline $11 / 03 / 1812: 01$ & 27.1 & 66.9 & 164.28 \\
\hline $11 / 03 / 1812: 01$ & 27.1 & 67.3 & 194.21 \\
\hline $11 / 03 / 1812: 01$ & 27.3 & 67.3 & 209.28 \\
\hline
\end{tabular}

Table 1: Example of the BtM output.

\section{Discussion}

To our knowledge, this is the first time that a datalogger to measure temperature, humidity, and conductance simultaneously as a proxy of the water content of poikilohydric organisms has been designed based on an open-access platform. The BtM datalogger is easy to build and costeffective, and also provides high-quality measurements of air humidity, temperature, and impedance data using minimal power.

The simple assembly is one of the main advantages of this datalogger. As it is an open-source project, we provide the data-logging software and a detailed scheme of its structure, together with a nontechnical manual for building a ready-to-use BtM datalogger. This makes the method accessible to any research group, even to those that do not work with an engineer or specialized technicians. Besides, the assembly of each datalogger requires just about 1 hour if the printed board circuit is used and about 4 hours if the circuit is mounted by the researchers. Additionally, the BtM datalogger is highly cost-efficient. The estimated cost of the components of each unit is approximately 100 euros, a fairly low price that can be reduced even further in large-scale projects by assembling batches of several dataloggers.

Although there have been several recent methodological developments aimed at implementing devices that measure different aspects related to the physiological activity of nonvascular cryptogam communities, the BtM fills an important knowledge gap. Raggio et al. ${ }^{15}$ employ Moni- 
$\mathrm{Da}$, a monitoring system that obtains physiological and microclimatic information. The physiological activity is collected through chlorophyll a fluorescence, a method widely used in the laboratory to estimate the activity of photosynthetic organisms. Although this method is highly accurate, it is significantly more expensive than the BtM datalogger. Besides, the monitoring system is a private company product, which cuts back the autonomy of the research group.

The two other methods that have recently been published are also based on estimating water content of nonvascular cryptogams. The first is based on thermal measurements (a dual-probe heat pulse (DPHP) method). Although promising results have recently been shown by Young et al. $^{16}$, the lack of any specific scheme in the paper makes assembling it without specialized knowledge highly challenging. Lastly, Weber et al. ${ }^{14}$ presented a sensor called the biocrust wetness probe (BWP), which is very similar to the BtM datalogger. However, they do not provide any scheme for its construction, which hinders the possibility of building the datalogger without the assistance of a specialist. We overcome this issue by providing not only the construction scheme but also the circuit board to assemble the datalogger. Interestingly, the BtM can be easily modified to measure biocrusts, isolated individuals, or cushions, just by changing the crocodile clips (for lichen or bryophyte individuals/cushions) to copper alloy electrode pins (for the biocrusts). If necessary, only part of the crocodiles can be replaced, allowing direct comparisons between the two measurement probe types.

When interpreting the results, the relationship between activity and water content should be carefully addressed, because the BtM does not directly measure photosynthesis. Photosynthesis and activity are closely related in nonvascular cryptogams since a dry poikilohydric organism is in metabolic cease and a wet one is active. However, the degree of photosynthetic activity cannot be inferred directly from the water content, even though a higher metabolic activity - and, thus, a higher photosynthetic activity - can be expected in a well-hydrated organism.

\section{Critical Steps:}

Despite the ease of assembly, there are some critical steps in the protocol that should be carefully addressed by researchers when mounting the sensor. First, as emphasized in the protocol, it is quite easy to produce short circuits when soldering, which, in the worst case, could result in serious damages to the microcontroller. It is very important to check for their presence with a multimeter and to remove them before connecting the batteries. We recommend using the provided PCB design since it significantly simplifies the process and may be the best option to overcome this issue. Second, not all IDE versions are compatible with the libraries required for this datalogger. It is important to download the proper one (1.0.6) to avoid any compatibility issues. Third, it is important to notice the polarity of the batteries. A polarity inversion could result in serious damages to the hardware. Fourth, calibration is a critical step. The BtM datalogger is designed so that the higher resolution coincides with the moment in which the cryptogam goes from dry to wet state. This implies that the conductance values saturate long before the sample is saturated in water. However, if the study at hand requires a higher accuracy around other values, it can be modified. Measures beyond one order of magnitude from this reference require the resistor to be changed and a recalibration process (see below). As the environmental temperature can affect the accuracy of the measurements, we recommend taking into account this factor when calibrating. To do so, the calibration should be done at low temperatures to check for changes in the measurement accuracy and stability (see Coxson ${ }^{12}$ for temperature effects).

\section{Modifications:}

Although most of the components of the BtM are fixed, some can be easily modified in the field without resoldering. The simplest modification is to replace the crocodile clips for other probe or measurement systems. For example, instead of the crocodile clips, a probe with two pins, such as the one suggested in Weber et al. ${ }^{14}$, can be used.

In remote environments, where changing the batteries may not be possible within the needed frequency, batteries could be complemented with a solar panel to power the BtM datalogger for longer periods.

By changing the reference resistors employed to measure the conductance, the rank of higher resolution can be easily modified to higher or lower values. If modified, we highly recommend a precise recalibration. Also, in the source code, the RValue variable, which is programmed for a resistor value of $330 \mathrm{~K} \Omega$, must be assigned to the new corresponding value (datalog.ino).

\section{Conclusion:}

Nonvascular cryptogam communities are highly diverse and play a number of different key ecological roles, so understanding their relationships with the abiotic environment is a crucial issue. The BtM datalogger has several applications that will help advance the knowledge of these relationships. For example, it will help deepen insights about the conditions where these organisms are acting as either carbon sinks or carbon sources. The fluctuations between these two roles are strongly related to abiotic conditions such as temperature and moisture ${ }^{3}$, but large amounts of data are needed to describe and understand the variations of that relationship at a global scale. This requires dense sensor networks that are possible only if they rely on low-cost and easy-to-implement equipment.

To summarize, this device is a useful tool for ecological research groups and overcomes the technical constraints of designing and building a datalogger. The combination of these two factors may lead to a popularization in the use of dataloggers to measure the water relations of nonvascular cryptogams in situ. This, in turn, can boost the establishment of medium and long-term monitoring networks. Developing these networks is essential to assessing the response of nonvascular cryptogams to local and regional environmental factors, as well as to determine their role in ecosystem processes (e.g., nutrient cycles, community assembly) and their most likely response in light of the changes on climatic and anthropic factors associated with global change.

\section{Disclosures}

The authors have nothing to disclose.

\section{Acknowledgments}

The authors are thankful to Manuel Molina (UAM) and Cristina Ronquillo (MNCN-CSIC) for the help provided during the calibration tests, and to

Belén Estébanez (UAM) for her help during the sampling campaigns. 


\section{References}

1. Fontaneto, D., Hortal, J. Microbial Biogeography: Is Everything Small Everywhere? In Microbial Ecological Theory: Current Perspectives. Edited by Ogilvie, L.A., Hirsch, P.R., 87-98, Caister Academic Press. Norfolk, UK (2012).

2. Proctor, M.C.F. et al. Desiccation-tolerance in bryophytes: a review. The Bryologist. 110 (4), 595-621 (2007).

3. Lindo, Z., Gonzalez, A. The Bryosphere: An Integral and Influential Component of the Earth's Biosphere. Ecosystems. 13 (4), 612-627 (2010).

4. Elbert, W. et al. Contribution of cryptogamic covers to the global cycles of carbon and nitrogen. Nature Geoscience. 5, 459-462 (2012).

5. Magill, R.E. Moss diversity: New look at old numbers. Phytotaxa. 9 (1), 167-174 (2014).

6. Söderström, L. et al. World checklist of hornworts and liverworts. PhytoKeys. (59), 1-828 (2016).

7. Lücking, R., Hodkinson, B.P., Leavitt, S.D. The 2016 classification of lichenized fungi in the Ascomycota and Basidiomycota - Approaching one thousand genera. The Bryologist. 119 (4), 361-416 (2016).

8. Bowker, M.A. Biological Soil Crust Rehabilitation in Theory and Practice: An Underexploited Opportunity. Restoration Ecology. 15 (1), $13-23$ (2007).

9. Wilske, B. et al. The $\mathrm{CO} 2$ exchange of biological soil crusts in a semiarid grass-shrubland at the northern transition zone of the Negev desert, Israel. Biogeosciences Discussions. 5 (3), 1969-2001 (2008).

10. Wardle, D.A. et al. Linking vegetation change, carbon sequestration and biodiversity: insights from island ecosystems in a long-term natural experiment. Journal of Ecology. 100 (1), 16-30 (2012).

11. Lindo, Z., Nilsson, M.-C., Gundale, M.J. Bryophyte-cyanobacteria associations as regulators of the northern latitude carbon balance in response to global change. Global Change Biology. 19 (7), 2022-2035 (2013).

12. Coxson, D.S. Impedance Measurement of Thallus Moisture Content in Lichens. The Lichenologist. 23 (1), 77-84 (1991).

13. Raggio, J. et al. Continuous chlorophyll fluorescence, gas exchange and microclimate monitoring in a natural soil crust habitat in Tabernas badlands, Almería, Spain: progressing towards a model to understand productivity. Biodiversity and Conservation. 23 (7), 1809-1826 (2014).

14. Weber, B. et al. Development and calibration of a novel sensor to quantify the water content of surface soils and biological soil crusts. Methods in Ecology and Evolution. 7 (1), 14-22 (2016).

15. Raggio, J. et al. Metabolic activity duration can be effectively predicted from macroclimatic data for biological soil crust habitats across Europe. Geoderma. 306, 10-17 (2017).

16. Young, M.H., Fenstermaker, L.F., Belnap, J. Monitoring water content dynamics of biological soil crusts. Journal of Arid Environments. 142 , 41-49 (2017)

17. Accurate low power temperature/relative humidity data logger. http://www.osbss.com/tutorials/temperature-relative-humidity/ (2015).

18. GitHub - united-ecology/btmboard. https://github.com/united-ecology/btmboard (2018).

19. Arduino - Software. https://www.arduino.cc/en/Main/Software (2018). 